\title{
On external influences on the radioactive decay rate fluctuations
}

\author{
Victor A. Panchelyuga, Maria S. Panchelyuga, Olga Yu. Seraya \\ victor.panchelyuga@gmail.com \\ Institute of Theoretical and Experimental Biophysics of Russian Academy of Science, Pushchino, Russia
}

The evolution of views on the possibility of external influence on the process of radioactive decay is briefly presented. Such an effect can lead to the appearance of periods in the time series of the radioactive decay rate fluctuations, which have been the subject of intensive study in the last decade. Two mechanisms for identifying periods are considered: the study of deviations from the theoretical curve of the radioactive decay law and the study of the properties of fluctuations. It is shown that the latter method leads to a universal spectrum of periods observed not only in the time series of the radioactive decay rate fluctuations, but also in the time series of fluctuations of processes of various nature. The main object of our study are periods in the radioactive decay rate fluctuations. The presence of such periods suggests the possibility of external influence on the process of radioactive decay. Therefore, we briefly consider the evolution of views on the possibility of such an effect. To do this, we distinguish several stages. The division into stages is only partially historical, but, mainly, each stage characterizes a certain ideas that is implemented in it.

Key words: radioactive decay; fluctuations; local fractal analysis; all permutations method.

\section{Introduction}

The first stage begins almost from the discovery of radioactivity by A. Becquerel in 1896 . Since that time, attempts of external influences on the radioactive decay rate have been made. As an example of such studies of that time, one can take Rutherford experiment [1], in which a certain amount of radioactive gas of radon was contained in a high pressure vessel with smokeless powder. Estimates show that at the moment of detonation of the explosive, the maximum temperature in the vessel reached $2500{ }^{\circ} \mathrm{C}$ and a pressure of about $1000 \mathrm{~atm}$. Under these conditions, the gamma activity of the radon remained unchanged. Finally, the results of such studies led to the conclusion that the rate of radioactive decay is constant under any conditions [2].

Studies of radon activity allowed Rutherford to obtain the basic law of radioactive decay [3]:

$$
N(t)=N_{0} e^{-\lambda t}
$$

where $N_{0}$ is the number of radioactive nuclei at an arbitrary initial moment $t=0, \lambda$ is the radioactive decay constant (characterizes the probability of one atom decay in one second). This law is valid only for the statistical mean and in the case of a large number of atoms in the radioactive isotope under examination. Fig. 1a) shows dependence (1). As can be seen from the figure, this is a smooth curve, which is completely determined by expression (1). The only parameter, on which the shape of the curve depends, is the decay constant $\lambda$, a constant which characterizes a given isotope.

However, in reality, the results of successive measurements of the radioactive decay rate, looks like in fig. 1b). These are random fluctuations. Each point of the curve in fig. 1a) is the mean calculated on the basis of the time series segment similar to that shown in fig. $1 \mathrm{~b}$ ).

The probability to detect the decay of $n$ particles from the total number $N$ of radioactive nuclei is:

$$
\omega_{n}=\frac{(N \lambda t)^{n}}{n !} \exp (-N \lambda t)
$$

Expression (2) is the Poisson distribution well known from statistics. As one can see, this statistical model lacks physical parameters that could describe an external influence.
The negative results of Rutherford's experiments on the effect on the radioactive decay rate have consolidated the opinion that radioactive decay, in terms of its instantaneous values, is a random and unpredictable process. This "random face" of radioactive decay is shown in fig. 1 and given by expression (2). On the other hand, if we determine with high accuracy the mean values of the radioactive decay rate, then it can serve as an analogue of ultra-stable clocks whose course is determined only by the decay constant $\lambda$, which does not depend on any external influences, as follows from (1), and is shown in Fig. 1a).

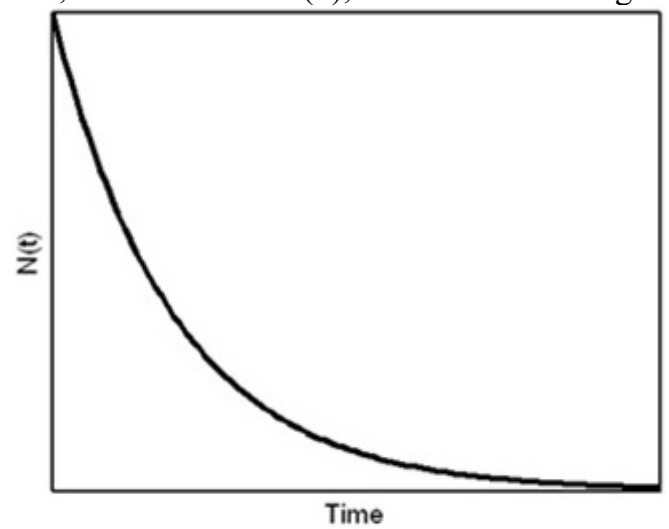

a)

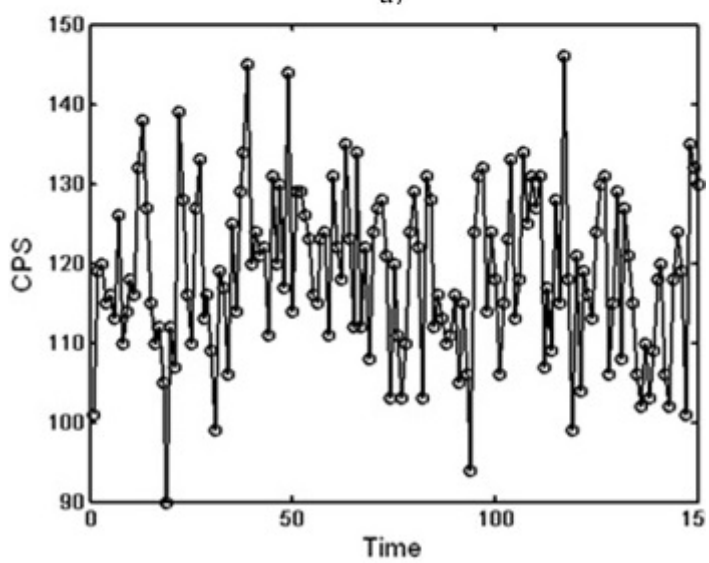

b)

Fig. 1. Two "faces" of radioactive decay: a deterministic, ultrastable process, a); a noise-like process, the instantaneous values of which are random and unpredictable, b). In fact, b) is a microscopic, local part of curve a) 


\section{2. "Deformation" of the electron shell and the radioactive decay rate}

The description of radioactive decay on the basis of (1) and (2), despite the fact that it was created in the initial period of the study of radioactivity, was included in most university textbooks, became the basis and, mainly, the only content, of the modern educational standard. At that, the authors usually emphasize that radioactive decay is a fundamentally statistical phenomenon, and that "Experiments with radioactive substances have shown that no external conditions (heating to high temperatures, magnetic and electric fields, high pressures) can affect the nature and decay rate" $[4$, p. 92]. That is, the possibility, at least hypothetical, of an external influence on the radioactive decay rate is completely excluded.

It is necessary to note that the first stage considered above is a pre-quantum one. The appearance and development of quantum mechanics marks the second stage, which begins in the post-war period and is characterized by the creation of quantum-mechanical models of nuclear phenomena. Due to this, it was shown that various effects on the electron shell (superstrong magnetic fields, pressure, changes in the chemical environment, degree of atomic ionization) leading to its changes or 'deformations' can affect the radioactive decay rate.

The findings of the aforementioned models were confirmed by numerous, carefully performed experiments. As an example is work [5], in which the process of $\beta$-decay of fully ionized ${ }^{187} \mathrm{Re}$ was studied. Full ionization reduced the half-life by $10^{9}$ times $\left(4.2 \cdot 10^{10}\right.$ years for a neutral atom and 33 years for a fully ionized one). In the case of $\alpha-$ decay, the $\alpha$-particle birth probability in the nucleus is associated with the problem of tunneling through the barrier. It was shown [6] that the permeability of a barrier depends on an electron shell. Therefore, by influencing the electron shell, one can change the rate of $\alpha$-decay. Thus, the Rutherford taboo on the possibility of influencing the rate of radioactive decay was canceled at the second stage.

Summing up, it can be noted that the second stage is characterized by the creation of theoretical models that demonstrate the possibility of influencing the rate of radioactive decay through a change in the state of the electron shell of an atom. The conclusions of these models are confirmed by numerous experiments. There are many works on this topic that are published in leading world physical journals.

\section{Periods in time series of the radioactive decay rate}

In contrast to the second stage, where the characteristic energy of the impact on the radioactive isotopes, is much less than the Coulomb barrier, but still very significant, in the third stage, as a rule, this energy is close to zero. In this case, both the isotope and the recording equipment are shielded from any external influences, which are usually of geophysical origin (temperature, pressure, humidity, etc).

It is very interesting that in such undisturbed time series, various periods were found. By the analogy with two 'faces' of radioactive decay, the works of the third stage, searching for the periods, study the deviations from the radioactive decay curve, fig. $1 a$ ). That is, the studied 'signal' is the difference between the theoretical curve (1) and the correspondingly averaged experimental time series. Subsequently, such time series of the differences are examined for the presence of periods by various methods of spectral and correlation analysis.

One of the first studies in this direction was a series of works [7-8], in which variations in the decay rate of ${ }^{14} \mathrm{C}$ and tritium with annual periods were found, as well as a noticeable effect of geomagnetic disturbances (strong magnetic storms). Also in [7], cyclical changes in the average and dispersion values of the decay rate of the measured tritium standard with periods of about 60 minutes and 3 hours were found. The authors emphasize that the amplitudes of the periods increase at low values of geomagnetic activity.

Perhaps, the most systematic study of periods in longterm series of the radioactive decay rate was carried out in the works of A.G. Parkhomov, started back in the 1990s and resulted by 2004 with convincing experimental evidence of the presence of various periods from diurnal to annual in $\beta$-radioactivity [9]. His studies are summarized in monograph [10].

Based on the work of A.G. Parkhomov and other authors one can conclude that annual and seasonal periods usually have relative amplitudes of $\sim 10^{-1} \ldots 10^{-3}$, while the amplitudes of the monthly and daily periods are $\sim 10^{-3} \ldots 10^{-5}$.

The annual period was also found in [11], where longterm series of the decay rate of the ${ }^{226} \mathrm{Ra}$ isotope (record length 15 years), measured in Physikalisch-Technische Bundesanstalt (PTB) and ${ }^{32} \mathrm{Si}$ (record length - 4 years), measured in Brookhaven National Laboratory (BNL), were analyzed. In addition to the annual period, it was also found that cyclical changes in the activity of ${ }^{226} \mathrm{Ra}$ and ${ }^{32} \mathrm{Si}$ correlate with a value of $1 / \mathrm{R}^{2}$, where $\mathrm{R}$ is the Earth-Sun distance, and demonstrate synchronism in the coincident part of the BNL and PTB time series.

Although the works of E. Fischbach are not pioneering (the annual period was discussed, for example, in [9]), they were destined to attract and, to some extent, change the world scientific opinion regarding the possibility of the existence of the abovementioned periods. Possibly, due to these works, the topics related to periods in the radioactive decay rate are actively discussed on the pages of international physics journals. To date, the number of such publications is in the hundreds.

Summarizing this section, we can conclude that the presence of periods may indicate the presence of some unidentified, presumably very weak external influence that affects the radioactive decay rate. At present, mechanisms of such influence can be only hypothesized.

\section{Periods in the time series of the radioactive decay rate fluctuations}

As noted in the previous section, in almost all the works of the third stage, where periods from the day-year range were found, classical methods of spectral and correlation analysis are used. This is due to the fact that the object of study in these works is the mean values slight variations on the curve of radioactive decay, fig. 1a). In the works that we refer to the fourth stage, the object of 
study is the time series of fluctuations in the radioactive decay rate, i.e., the process shown in fig. $1 b$ ). It this case the properties of fluctuations are investigated, without respect to behavior of average values. In this case, weak variations of the averages can be eliminated from the studied time series by the appropriate computational procedure, but this does not affect the information contained in the fluctuations.

To study the properties of fluctuations, time series similar to those shown in fig. 1b) must be parameterized in a certain way. After that, the appropriate methods are used to analyze the obtained sequences of parameters. Below we will briefly consider two groups of works that differ in the method of parameterization: the expert histogram comparisons method of S.E. Schnoll [12] (parameterization is performed using smoothed histograms constructed from short (30-60 points) consecutive segments of time series) and local fractal analysis by the all permutations method [13-14].

\section{S.E. Schnoll research}

These studies started in 1951-56, when S.E. Schnoll began a systematic study of fluctuations in the measurement results of the biochemical reactions rates. The reason to start these studies was an unusually large amplitude of observed fluctuations. However, their most striking property was strongly rugged histograms shapes spectra of amplitudes of the measured fluctuations values. In some experiments, these rugged histograms were surprisingly similar to each other. This phenomenon was called the macroscopic fluctuations phenomenon.

Using the histogram method (the method of time series studying by the pairwise expert comparison of the shapes of histograms [12]), the following basic properties of the macroscopic fluctuations phenomenon were obtained [1314].

A near-zone effect. A significantly higher probability of the appearance of similar shapes of histograms in the nearest (neighboring) non-overlapping intervals of the time series.

Universality of the macroscopic fluctuations phenomenon. It lies in the high similarity in the shape of histograms constructed from the results of simultaneous, independent measurements of fluctuations in processes of various nature.

Periodicity in manifestation of the macroscopic fluctuations phenomenon. An important evidence of the nonrandomness of the shapes of histograms is their regular changes in time. These changes of the patterns of histograms are manifested in the presence of periods: diurnal (1440 min and $1436 \mathrm{~min}$ ), about 27-days periods, annual periods ('calendar' - 365 days and sidereal - 365 days 6 hours and 9 minutes).

A local time effect. Manifests itself in a high probability of occurrence of pairs of histograms with similar shapes in different geographical locations at the same local (longitude) time.

Dependence of the similarity of the shapes of histograms on the direction in space.

Specific histogram shapes, which appear at the moments of new moons and at the maximum of solar eclipses, during the rising and setting of the Sun and the Moon.

\section{Local fractal analysis by all permutations method}

In [15-16], a local fractal analysis of noise-like time series by the all permutations method (APM) was developed. This method synthesized the basic ideas of the method of minimum cover [33] (MMC) with the requirement of fractal dimension invariance with respect to linear transformations (shifts, dilatations, mirror reflections), as well as regarding to permutations of the elements of a time series segment, on the basis of which the fractal dimension is calculated. The latter property is a distinctive feature of the APM-method, which gives it a number of unique properties. The most important of them is locality - the ability to calculate the fractal dimension for short (tens of points) segments of the analyzed time series.

Unlike to the MMC-method, for a time series segment of length $N=2^{n}$, the APM-method allows one to analyze $N-1$ scales, rather than $n$, as in the MMC-method and other methods commonly used to calculate fractal dimension. Due to this property, the value of $N$ can be significantly reduced, and the accuracy of determining the fractal dimension increases significantly.

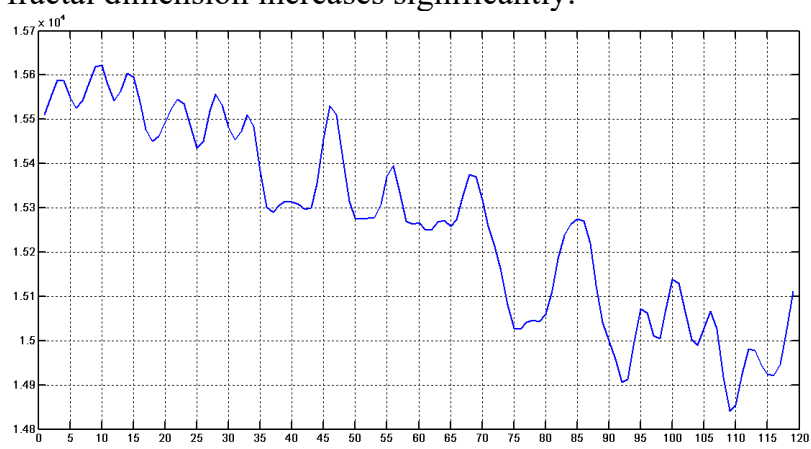

Fig. 2. Sum of 329-day interval distributions

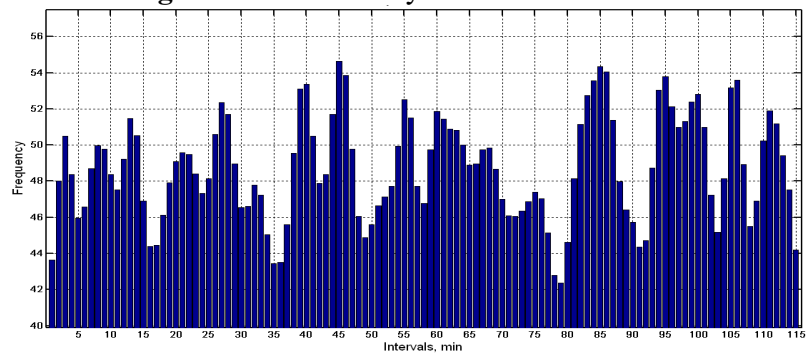

Fig. 3. Probability distribution of peak occurrences. Based on 329-day interval distributions containing 5695 peaks

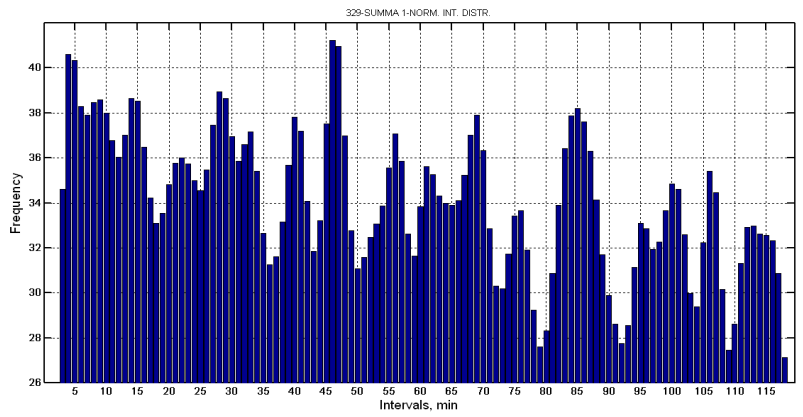

Fig. 4. Probability distribution of peak occurrences. Based on 329-day normalized distribution of intervals containing 5695 peaks 


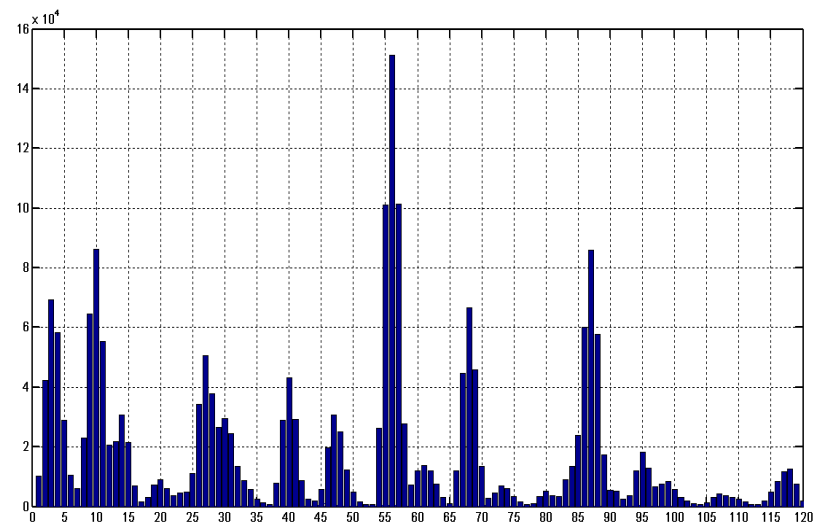

Fig. 4. Multiplication of 329-day normalized interval distributions

One of the first results of the APM-method application for the analysis of noise-like time series is the study of a 329 -day array of fluctuations in the $\alpha$-decay rate, as a result of which a stable set of periods was found in the range of 1-115 $\mathrm{min}$ [17-18]. It was shown that the periods found in [17-18] coincide with the periods of the Earth natural oscillations [19]. This coincidence is manifested for both classical and long-wave oscillations of the Earth [20].

For the indicated range of periods, not only the close relationship of the found spectrum with the spectrum of the Earth's natural oscillations was shown, but also its universal nature: the spectra of the periods found for fluctuation process in the systems of various nature (physical, chemical, biological) always coincided with the corresponding part of the spectrum found for time series of the $\alpha$-decay rate fluctuations.

In further studies, using the APM-method, stable ultradian periods were obtained in the range of 2-24 hours [21-22], which also coincided with the corresponding periods of the Earth's natural long-wave oscillations [23].

\section{Conclusions}

The previous studies [17-18, 21-22], have shown that most periods from the found spectrum coincide with periods of the Earth's natural oscillations. Its universal nature was shown as well: the periods found in the time series of fluctuations of various nature processes always coincide with the corresponding part of this spectrum. Further investigations made it possible to clarify a number of periods of the found spectrum, as well as to reveal new ones, including those not belonging to the spectrum of the Earth's natural oscillations [24]. Along with the coincidence of the spectrum with the spectra of various fluctuation processes of 'terrestrial' origin, for example, temperature fluctuations of small mammals and birds [25] and planaria chemiluminescence [26], its coincidence with the spectra of periods of a number of astrophysical systems is very interesting.

Thus, the spectrum of periods [17-18] was also found in the spectra of astrophysical masers [27-30] and in the spectrum of rotational periods of asteroids [31]. All previously mentioned allows us to make an assumption about the cosmophysical origin of the spectrum [17-18]. It is possible that in this case we are dealing with a global synchronization to which, according to A.M. Molchanov hypothesis [32], any dynamically mature system should come.

In view of the above-said, it is important to note the universality of the spectrum [17-18], which can be found in fluctuations of various nature processes.

\section{References:}

[1] E. Rutherford Radioactive Substances and Their Radiations. Cambridge University Press, New York, 1913

[2] S. E. Rutherford, J. Chadwick, and C. Ellis Radiations from Radioactive Substances. Cambridge University Press, 1930.

[3] Mukhin K.N. Experimental nuclear physics. The physics of the atomic nucleus. Part 1. Properties of nucleons, nuclei and radioactive radiation. Moscow, Energoatomizdat, 1993 - 376 p.

[4] Rakobolskaya I.V. Nuclear physics. Moscow, MSU, 1971 - 295 c.

[5] Bosch F., Faestermann T., Friese J., et al. Observation of bound-state $\beta^{-}$-decay of fully ionized ${ }^{187} \mathrm{Re}$ : ${ }^{187} \mathrm{Re}-{ }^{187}$ Os cosmochronometry // Phys. Rev. Lett., 1996, V. 77, No.26, pp.5190-5193.

[6] Victor A. Erma Electron effects on barrier penetration // Phys. Rev., 1957, V.105, No.6, pp.1784-1787.

[7] Avdonina E.N., Lukyanov V.B. Heliogeophysical effects in the results of measuring radioactivity by liquid scintillation counting methods and statistics of radioactive decay // Biophysics, 1995, v.40, No.4, pp. 876-881.

[8] Avdonina E.N., Lukyanov V.B. // Dep. VINITI, № 2492-889, 18.04.1989.

[9] A.G. Parkhomov, Ye.F. Maklyayev Investigation of rhythms and fluctuations during long-term measurements of radioactivity, frequency of quartz resonators, noise of semiconductors, temperature and atmospheric pressure. // Fizicheskaya mysl' Rossii, No. 1, 2005.

[10] Parkhomov A.G. Space. Earth. Person. New aspects of science. Moscow, 2009 - 272 c.

[11] Jere H. Jenkins, Ephraim Fischbach, John B. Buncher, John T. Gruenwald, Dennis E. Krause, Joshua J. Mattes Evidence of correlations between nuclear decay rates and Earth-Sun distance // Astroparticle Physics 32 (2009) 42-46.

[12] S.E. Schnol, V.A. Panchelyuga The phenomenon of macroscopic fluctuations. Methods of measurement and processing of experimental data. // Mir izmereniy, 2007, No.6, pp. 49-55.

[13] Shnoll S.E. Cosmophysical Factors in Stochastic Processes. American Research Press, Rehoboth (NM), 2012 - 433p.

[14] Shnoll S.E., Kolombet V.A., Pozharskii E.V., Zenchenko T.A., Zvereva I.M., Konradov A.A. Realization of discrete states during fluctuations in macroscopic processes // Physics Uspekhi, 1998, 41 pp. 1025-1035.

[15] V.A. Panchelyuga, M.S. Panchelyuga Fractal Dimension and Histogram Method: Algorithm and Some Preliminary Results of Noise-Like Time Series 
Analysis // Biophysics, 2013, Vol.58, No.2, pp.283289.

[16] V.A. Panchelyuga, M.S. Panchelyuga Local fractal analysis of non-stationary time series by all permutations method // Hypercomplex numbers in geometry and physics, 2014, Vol.11, No.1(21), pp.107-133.

[17] Panchelyuga V.A., Panchelyuga M.S. Local Fractal Analysis of Noise-Like Time Series by the AllPermutations Method for 1-115 min Periods // Biophysics, 2015, Vol. 60, No. 2, pp. 317-330.

[18] V.A. Panchelyuga, M.S. Panchelyuga Some preliminary results of local fractal analysis of noiselike time series by all permutations method for periods 1-120 min // Hypercomplex numbers in geometry and physics, 2014, Vol.11, No.1(21), pp. 134-156.

[19]T.G.Masters, R.Widmer Free oscillations: frequencies and attenuations. In Global Earth physics: a handbook of physical constants / Ed. by Thomas J. Ahrens, American Geophysical Union, 1995, pp.104125.

[20] Petrova L.N. Seismogravitational oscillations of the Earth and possible mechanism of their formation // Biophysics, 1992, Vol. 37, No. 3, pp. 508-516.

[21] V.A. Panchelyuga, M.S. Panchelyuga, O.Yu. Seraya Preliminary periods investigation in time series of alpha-decay rate fluctuations // Hypercomplex Numbers in Geometry and Physics, 2016, Vol. 13, No. 2(25), pp. 211-216.

[22] V.A.Panchelyuga, M.S.Panchelyuga, O.Yu.Seraya Preliminary results of the study of the relationship between ultradian periods (2-24 hours) in the time series of alpha decay rate fluctuations with the Earth's natural oscillations // Planet Earth System, Moscow, LENAND, 2017, pp.227-232.

[23] Barkin Yu.V. Free Translational Oscillations of The System "Core-Mantle" of the Earth and Variations of Natural Processes with Hour Periods // Nonlinear world, 2007, v.5, No.1-2, pp.101-109.

[24] Panchelyuga V.A., Vladimirsky B.M., Panchelyuga M.S., Seraya O.Yu., Panikhin V.A. Periods of 50, 80 and $160 \mathrm{~min}$ in time series of alpha-decay rate fluctuations // Proceedings of the XXI Russian Conference "Solar and Solar-Earth Physics - 2017", St. Petersburg, Pulkovo, October 10-14, 2017, p. 261264.

[25]M.E. Diatroptov, V.A. Panchelyuga, M.S. Panchelyuga. On the coincidence of the spectrum of periods in the time series of temperature fluctuations of starlings and rats with the spectrum of periods of fluctuations in the rate of alpha decay // Proceedings of the XV International Conference "Finsler Extension of Relativity Theory" (FERT-2019) / Eds.: Pavlov D.G., Panchelyuga V.A. - Moscow, 11th format, 2019 - pp.30-35.

[26] Panchelyuga V.A., Tiras Kh.P., Novikov K.N., Panchelyuga M.S., Nefedova S.E. On the coincidence of the spectrum of periods in the time series of chemoluminescence of planaria with the spectrum of periods found in the time series of fluctuations in the alpha decay rate // Proceedings of the XV International Conference "Finsler Extension of
Relativity Theory" (FERT-2019) / Eds.: Pavlov D.G., Panchelyuga V.A. - Moscow, 11th format, 2019 pp.36-41

[27] S.Siparov, V.Samodurov, and G.Laptev Origin of observed periodic components in astrophysical masers' spectra // Monthly Notices of the Royal Astronomical Society, 2017, 467, pp. 2813-2819.

[28] V.A. Panchelyuga, M.S. Panchelyuga On the relationship between the spectrum of periods in the time series of alpha decay rate fluctuations and periodic components in the spectra of astrophysical masers // Proceedings of the XIII International Conference "Finsler Extension of Relativity Theory" (FERT-2017) / Eds.: Pavlov D.G., Panchelyuga V.A. - Moscow, 11th format, 2017 - pp. 69-70.

[29] Panchelyuga V.A., Vladimirsky B.M., Panchelyuga M.S. On the coincidence of the spectrum of periods in the time series of alpha decay rate fluctuations with periodic components in the astrophysical masers spectra // Planet Earth System, Moscow, LENAND, 2019, pp.115-118.

[30] Panchelyuga V.A., Vladimirsky B.M., Panchelyuga M.S. On some possible mechanisms of the relationship between the spectrum of periods in the time series of alpha decay rate fluctuations and periodic components in the spectra of astrophysical masers // Proceedings of the XIV International Conference "Finsler Extension of Relativity Theory" (FERT-2017) / Eds.: Pavlov D.G., Panchelyuga V.A. - Moscow, 11th format, 2018 - pp.89-91.

[31]V.A. Panchelyuga, M.S. Panchelyuga On the coincidence of the spectrum of periods in the alpha decay rate fluctuations with the spectrum of rotational periods of asteroids // Proceedings of the XV International Conference "Finsler Extension of Relativity Theory" (FERT-2019) / Eds.: Pavlov D.G., Panchelyuga V.A. - Moscow, 11th format, 2019 pp.27-29.

[32] A.M. Molchanov The resonant structure of the Solar system. The law of planetary distances // Icarus, 1968, Vol.8, No.1/3, pp.203-215.

[33] M.M. Dubovikov, N.V. Starchenko, M.S. Dubovikov Dimension of minimal cover and fractal analysis of time series // Physica A, 339, 2004, pp. 591-608.

\section{About the authors}

Victor A. Panchelyuga, PhD (Physics and Mathematics), senior scientist in the Institute of Theoretical and Experimental Biophysics, RAS E-mail: victor.panchelyuga@gmail.com.

Maria S. Panchelyuga, scientist in the Institute of Theoretical and Experimental Biophysics, RAS, E-mail: victor.panchelyuga@gmail.com.

Olga Yu. Seraya, junior scientist in the Institute of Theoretical and Experimental Biophysics, RAS E-mail: olgaseraya@gmail.com 